\title{
Dynamic and Static Small-Animal SPECT in Rats for Monitoring Renal Function After ${ }^{177}$ Lu-Labeled Tyr ${ }^{3}$-Octreotate Radionuclide Therapy
}

\author{
Marleen Melis ${ }^{1}$, Jan de Swart ${ }^{1}$, Monique de Visser ${ }^{1}$, Saskia C. Berndsen ${ }^{1}$, Stuart Koelewijn ${ }^{1}$, Roelf Valkema ${ }^{1}$, \\ Otto C. Boerman ${ }^{2}$, Eric P. Krenning ${ }^{1}$, and Marion de Jong ${ }^{1}$ \\ ${ }^{1}$ Department of Nuclear Medicine, Erasmus MC, Rotterdam, The Netherlands; and ${ }^{2}$ Department of Nuclear Medicine, Radboud \\ University Medical Centre, Nijmegen, The Netherlands
}

High kidney radiation doses during clinical peptide receptor radionuclide therapy (PRRT) with $\beta$-particle-emitting radiolabeled somatostatin analogs will lead to renal failure several months after treatment, urging the coinfusion of the cationic amino acids lysine and arginine to reduce the renal radiation dose. In rat PRRT studies, renal protection by the coadministration of lysine was confirmed by histologic examination of kidney specimens indicating nephrotoxicity. In the current study, we investigated dedicated small-animal SPECT/CT renal imaging in rats to monitor renal function in vivo during follow-up of PRRT, with and without lysine. Methods: The following 3 groups of rats were imaged using a multipinhole SPECT/CT camera: controls (group 1) and rats at more than $90 \mathrm{~d}$ after therapy with $460 \mathrm{MBq}(15 \mu \mathrm{g})$ of ${ }^{177} \mathrm{Lu}$-DOTA-Tyr ${ }^{3}$-octreotate without (group 2) or with (group 3) a 400-mg/kg lysine coinjection as kidney protection ( $n \geq 6$ per group). At 90 and $140 \mathrm{~d}$ after therapy, static kidney scintigraphy was performed at $2 \mathrm{~h}$ after injection of $25 \mathrm{MBq}$ of $99 \mathrm{mTc}$-dimercaptosuccinic acid (99mTc-DMSA). In addition, dynamic dual-isotope renography was performed using $50 \mathrm{MBq}$ of ${ }^{111} \mathrm{In}$-diethylenetriaminepenta-

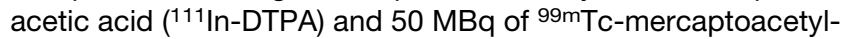
triglycine (99mTc-MAG3) at 100-120 d after therapy. Results: ${ }^{111} \mathrm{In}$-DTPA and 99mTc-MAG3 studies revealed a time-activity pattern comparable to those in patients, with a peak at 2-6 min followed by a decline of renal radioactivity. Reduced ${ }^{111}$ In-DTPA, 99mTc-MAG3, and 99mTc-DMSA uptake indicated renal damage in group 2, whereas group 3 showed only a decrease of ${ }^{99 m}$ Tc-MAG3 peak activity. These results indicating nephrotoxicity in group 2 and renal protection in group 3 correlated with levels of urinary protein and serum creatinine and urea and were confirmed by renal histology. Conclusion: Quantitative dynamic dual-isotope imaging using both ${ }^{111}$ In-DTPA and $99 \mathrm{~m}$ Tc-MAG3 and static 99mTc-DMSA imaging in rats is feasible using small-animal SPECT, enabling longitudinal monitoring of renal function. 99mTc-MAG3 renography, especially, appears to be a more sensitive marker of tubular function after PRRT than serum chemistry or ${ }^{99 m T c-D M S A ~ s c i n t i g r a p h y . ~}$

Received Jun. 13, 2010; revision accepted Sep. 8, 2010.

For correspondence or reprints contact: Marleen Melis, Department of Nuclear Medicine, Erasmus MC,'s Gravendijkwal 230, 3015 CE Rotterdam, The Netherlands.

E-mail: m.melis@erasmusmc.nl

COPYRIGHT @ 2010 by the Society of Nuclear Medicine, Inc.
Key Words: animal imaging; radionuclide therapy; renal; PRRT; dynamic imaging; micro-SPECT; nephrotoxicity; renography

J Nucl Med 2010; 51:1962-1968

DOI: 10.2967/jnumed.110.080143

$\mathbf{R}$ ecent technical improvements of the resolution and sensitivity of dedicated small-animal SPECT cameras has opened new possibilities in preclinical research. Multipinhole collimated cameras achieve submillimeter spatial resolution, and accurate quantification of the amount of radioactivity in tissues is feasible $(1,2)$. Therefore, the noninvasive technique of in vivo functional imaging can be used to follow up longitudinal induction and progression of disease (3).

Peptide receptor radionuclide therapy (PRRT) in patients with somatostatin receptor-overexpressing neuroendocrine tumors using radiolabeled somatostatin analogs such as octreotate shows beneficial results. Significant tumor response, survival benefit, and improvement of quality of life are obtained (4). However, partial reabsorption of the radiopeptides in the kidneys may lead to long-term nephrotoxic effects, especially when ${ }^{90}$ Y-labeled analogs are administered $(5,6)$. Recent developments to further improve tumor response rates include salvage therapy and combined therapy with both ${ }^{90} \mathrm{Y}$ - and ${ }^{177} \mathrm{Lu}$-labeled radiopeptides, supporting the need for kidney protection (7-9). Today, coinfusion of lysine and arginine during PRRT is a protective measure in the clinic, resulting in a $35 \%-40 \%$ reduction of the kidney radiation dose $(10-12)$, also found in preclinical animal models (13). Therapy with 555 or 278 $\mathrm{MBq}$ of ${ }^{177} \mathrm{Lu}$-DOTA-Tyr ${ }^{3}$-octreotate resulted in complete remission (14) or $80 \%$ complete remission and $20 \%$ partial remission (15), respectively, of somatostatin receptorexpressing tumors in rats. PRRT with $555 \mathrm{MBq}$, however, delivers a $60-\mathrm{Gy}$ radiation dose to the kidneys, inducing renal toxicity beyond $90 \mathrm{~d}$ after therapy $(16,17)$.

In patients, renal function can be monitored by renography and measurements of serum creatinine and creatinine clearance as markers of glomerular filtration rate $(14,18)$. 
The uptake of ${ }^{99 \mathrm{~m}} \mathrm{Tc}$-radiolabeled dimercaptosuccinic acid ( ${ }^{99 m}$ Tc-DMSA) by the kidneys is directly related to tubular function. Although ${ }^{99 \mathrm{~m}} \mathrm{Tc}-\mathrm{DMSA}$ scintigraphy is clinically widely used to provide information on renal cortical morphology (19), the exact mechanism of renal handling is unknown. The 2 postulated mechanisms of ${ }^{99} \mathrm{~m}$ Tc-DMSA uptake are peritubular extraction or absorption of plasma protein-bound ${ }^{99 \mathrm{~m}} \mathrm{Tc}-\mathrm{DMSA}$ directly from the blood into the proximal tubular cells or glomerular filtration, followed by tubular reabsorption. Discussions on the relative contribution of each of these pathways are ongoing (20-26). Dynamic imaging of the clearance of radiolabeled diethylenetriaminepentaacetic acid (DTPA) monitors glomerular filtration, because this small molecule is cleared only via this route. ${ }^{99 \mathrm{~m}} \mathrm{Tc}$-labeled mercaptoacetyltriglycine $\left({ }^{99 \mathrm{~m}} \mathrm{Tc}-\right.$ MAG3) is primarily rapidly extracted from the blood; a $10 \%-15 \%$ fraction is also cleared via glomerular filtration. Extraction of ${ }^{99 \mathrm{~m}} \mathrm{Tc}-\mathrm{MAG} 3$ by basolateral uptake into cells lining proximal tubules is mediated by an active organic anion transport system, especially in the first convoluted part (S1 and S2) of the proximal tubules (27).

In the current study, dynamic dual-isotope imaging of ${ }^{111} \mathrm{In}-\mathrm{DTPA}$ and ${ }^{99 \mathrm{~m} T c-M A G 3}$ and static ${ }^{99 m}$ Tc-DMSA imaging (Fig. 1) were performed to study glomerular filtration, tubular secretion, and peritubular absorption in rats beyond $90 \mathrm{~d}$ after therapy with ${ }^{177} \mathrm{Lu}$-DOTA-Tyr ${ }^{3}$-octreotate PRRT, with or without kidney protection by lysine coadministration, and results were compared with those for untreated controls. For this purpose, the multipinhole collimated small-animal NanoSPECT/CT camera (Bioscan Inc.) was used (24). Data were correlated with urine and serum chemistry and kidney histology.

\section{MATERIALS AND METHODS}

\section{Animals}

Animal studies were conducted in accordance with the guidelines of the Animal Welfare Committee of our medical center. For all experiments, male Lewis rats (Harlan) were used ( $n=6-9$ per group). Body weight (BW) was determined twice a week up to $140 \mathrm{~d}$ after therapy. Urine samples were collected every $3 \mathrm{wk}$ after rats were administered PRRT in metabolic cages for $24 \mathrm{~h}$.

\section{Radionuclides, Peptide, Radiopharmaceuticals, and Chemicals}

${ }^{177} \mathrm{LuCl}_{3}$ was obtained from IDB Holland, and DOTA$\mathrm{Tyr}^{3}$-octreotate was obtained from BioSynthema. Radiolabeling was performed according to previously published procedures (28). A PRRT dose of $460 \mathrm{MBq}$ of ${ }^{177} \mathrm{Lu}$-DOTA-Tyr ${ }^{3}$-octreotate per $15 \mu \mathrm{g}$ was used. A fresh $400 \mathrm{mg} / \mathrm{mL}$ solution of L-lysine (Sigma) was prepared and coinjected with ${ }^{177} \mathrm{Lu} \mathrm{DOTA}-\mathrm{Tyr}^{3}{ }^{3}$ octreotate in a dose of $400 \mathrm{mg} / \mathrm{kg}$ of BW (13).

${ }^{111} \mathrm{InCl}_{3}$, DMSA, and MAG3 kits were purchased from Covidien; DMSA and MAG3 were labeled with ${ }^{99 \mathrm{~m}} \mathrm{Tc}$ according to the provided procedure. For ${ }^{99 \mathrm{~m}} \mathrm{Tc}-\mathrm{DMSA}$ and ${ }^{99 \mathrm{~m}} \mathrm{Tc}-\mathrm{MAG} 3$, the concentrations were 100 and $250 \mathrm{MBq} / \mathrm{mL}$, respectively. ${ }^{111} \mathrm{In}-\mathrm{DTPA}$ was prepared by the addition of $100 \mu \mathrm{L}$ of $4 \mathrm{mM}$ DTPA to 400 $\mathrm{MBq}$ of ${ }^{111} \mathrm{InCl}_{3}$ (pH 3.5-4) and adjusted with saline to a concentration of $250 \mathrm{MBq} / \mathrm{mL}$.

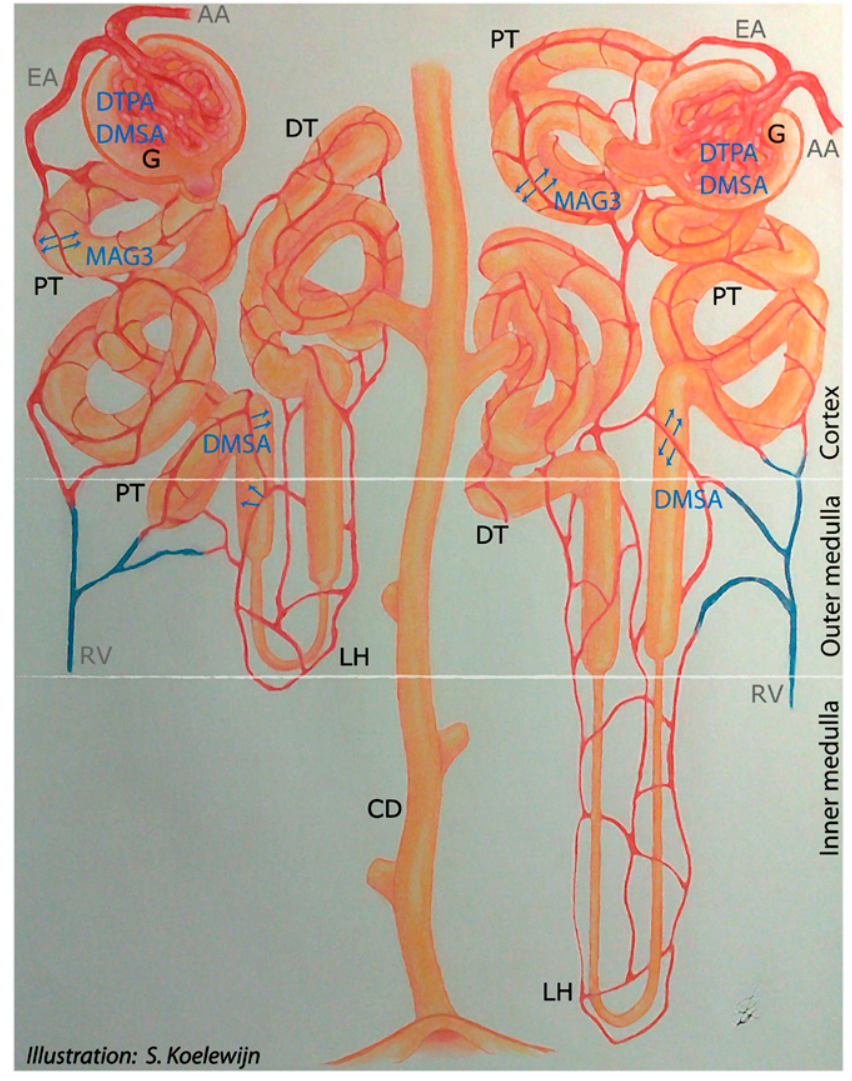

FIGURE 1. Blood enters functional units of kidneys, the nephrons, via afferent arteriole (AA) of renal artery. In the capillary network in glomerulus (G), waste and useful molecules are filtered from blood and transported into proximal tubules (PT). Waste molecules are excreted in primary urine via loop of Henle (LH) and distal tubules (DT) into collecting duct (CD) leading to renal pelvis and bladder. In first convoluted part of PT, megalin/cubilin receptors are expressed, playing a significant role in reabsorption of useful molecules. Extraction from blood in efferent arteriole (EA) (27) directly into PT is another route of excretion of molecules into urine, which is the most prominent way of ${ }^{99 m T C-M A G 3}$ clearance. ${ }^{111} \mathrm{In}$-DTPA is cleared only via glomerular filtration and is directly excreted into urine, whereas ${ }^{99 m T c-D M S A}$ is partly filtrated in glomeruli, followed by partial reabsorption in PT. 99mTc-DMSA is also partially peritubularly extracted from blood, primarily in last straight part of PT, which may extend into outer medulla of kidney. RV = renal vein.

\section{Molecular Imaging}

Rats were imaged using a 4-head multipinhole NanoSPECT/CT camera. The energy peak settings (window width $\pm 10 \%$ ) were 55, 113, and $208 \mathrm{keV}$ for ${ }^{177} \mathrm{Lu}, 171$ and $245 \mathrm{keV}$ for ${ }^{111} \mathrm{In}$, and $140 \mathrm{keV}$ for ${ }^{99 \mathrm{~m}} \mathrm{Tc}$. During ${ }^{111} \mathrm{In}$ and ${ }^{99 \mathrm{~m}} \mathrm{Tc}$ dual-isotope imaging, no visible cross-talk from the $\gamma$-rays emitted by ${ }^{111} \mathrm{In}$ into the 140 $\mathrm{keV}$ window was observed.

Nine pinhole apertures (diameter, $2.5 \mathrm{~mm}$ ) were used on each camera head, with a transaxial field of view of $60 \mathrm{~mm}$. On the basis of the CT topogram, the axial scan length used for all imaging procedures was $55 \mathrm{~mm}$ covering the renal region. Animals were imaged while anesthetized with isoflurane and $\mathrm{O}_{2}$, and body temperature was maintained using a heated bed.

Four days after therapy, rats were scanned to quantify the renal ${ }^{177} \mathrm{Lu}$ activity dose. ${ }^{99 \mathrm{~m}} \mathrm{Tc}-\mathrm{DMSA}$ (25 MBq, administered in 250 $\mu \mathrm{L}$ via the dorsal penis vein) scans were obtained at days 90 and 
140 after therapy, $2 \mathrm{~h}$ after injection. ${ }^{177} \mathrm{Lu}$-DOTA-Tyr ${ }^{3}$-octreotate and ${ }^{99 m}$ Tc-DMSA images were acquired for 12 min using 24 projections (60 s/projection per head).

Between 100 and $120 \mathrm{~d}$ after therapy, dual-isotope dynamic scans were obtained. Before scans were started, rats were anesthetized and a 27-gauge infusion set, with a tubing length of $30 \mathrm{~cm}$ (Venisystems; Hospira) filled with saline supplemented with heparin (50 international units $[\mathrm{IU}] / \mathrm{mL}$ ), was inserted into a tail vein. Immediately after the start of the scan, $200 \mu \mathrm{L}(50 \mathrm{MBq})$ of ${ }^{99 \mathrm{~m}} \mathrm{Tc}-\mathrm{MAG} 3$, followed by $200 \mu \mathrm{L}$ (50 MBq) of ${ }^{111} \mathrm{In}-\mathrm{DTPA}$, was administered and flushed with $700 \mu \mathrm{L}$ of saline (dead volume of catheter, $400 \mu \mathrm{L}$ ). Twenty scans of 2 min each (16 projections per head, $9 \mathrm{~s} /$ projection) were obtained, resulting in a total acquisition time of $40 \mathrm{~min}$. To study the influence of extrahydration on the efflux of ${ }^{99 m}$ Tc-MAG3 and ${ }^{111}$ In-DTPA from the kidneys, an additional group of control rats was imaged starting $45 \mathrm{~min}$ after the intraperitoneal injection of $5 \mathrm{~mL}$ of saline.

Quantification of the amount of radioactivity in a volume of interest over the kidneys was performed using InVivoScope software (Bioscan). Detected counts in the volume of interest were converted into megabecquerels using a correction factor obtained by scanning a water phantom with the same volume as a rat body to correct for attenuation and filled with a known amount of radioactivity. Radioactivity in the volume of interest over the whole kidneys, or over a cortical part in ${ }^{99 m}$ Tc-MAG3 images, was quantified in each of the 20 scans during renography to create a time-activity curve. Peak activity of ${ }^{99 \mathrm{~m}} \mathrm{Tc}-\mathrm{MAG} 3$ or ${ }^{111} \mathrm{In}$-DTPA was defined as the mean activity at 2-6 min after injection or 2-4 min after injection, respectively.

\section{Analytic Procedures}

Urinary protein content and serum urea and creatinine levels were measured as previously published (16). At day 90 after therapy, blood was drawn from a tail vein before injection of ${ }^{99 \mathrm{~m}} \mathrm{Tc}-$
DMSA, and at euthanasia at day 140 after therapy blood was collected to store serum.

\section{Ex Vivo Autoradiography and Histology}

As previously described, the localization of ${ }^{99 m} \mathrm{Tc}-D M S A$ in the kidneys was visualized by ex vivo autoradiography (17). The grade of renal damage was evaluated microscopically according to a scale from grade 0 (no damage) to 1 (little tubular dilation), 2 (basal membrane thickening), 3 (shrinkage of glomeruli, flat tubule epithelium) and 4 (severe tubular and glomerular damage), as described earlier in detail (16).

\section{Statistics}

Data were expressed as mean $\pm \mathrm{SD}$. Statistical analyses were performed using the Student $t$ test.

\section{RESULTS}

Quantification of ${ }^{177} \mathrm{Lu}$ in the kidneys at $4 \mathrm{~d}$ after therapy showed that $3.8 \pm 0.3 \mathrm{MBq}$ were retained in the kidneys of the unprotected animals, whereas $2.0 \pm 0.1 \mathrm{MBq}{ }^{177} \mathrm{Lu}$ localized in the kidneys of the lysine-protected rats (Table 1) - a significant reduction of renal retention of approximately $45 \%$. Irradiation of the renal tubular cells by the $\beta$-particles emitted by ${ }^{177} \mathrm{Lu}$ obviously led to renal problems, starting from $50 \mathrm{~d}$ after therapy in PRRT rats without lysine kidney protection. Compared with the normal BW increase over time in controls, BW stabilized in PRRT rats and urinary protein loss was detected. A further increase of protein loss in urine and rise of urea and creatinine serum levels determined at day 90 after therapy confirmed development of renal damage (Table 1). Histologic grading of

TABLE 1

Overview of Results in Control, PRRT, and PRRT + Lysine Rats

\begin{tabular}{|c|c|c|c|c|}
\hline Parameter & $\begin{array}{l}\text { Days after } \\
\text { therapy }\end{array}$ & $\begin{array}{c}\text { Control } \\
(P \text { control vs. PRRT) }\end{array}$ & $\begin{array}{l}\text { PRRT (P PRRT vs. } \\
\text { PRRT + lysine) }\end{array}$ & $\begin{array}{l}\text { PRRT + lysine (P control } \\
\text { vs. PRRT + lysine) }\end{array}$ \\
\hline${ }^{177}$ Lu uptake (MBq/kidney) & 4 & - & $3.8 \pm 0.3^{*}$ & $2.0 \pm 0.1$ \\
\hline \multirow[t]{2}{*}{ BW, \% vs. day 0} & 90 & $134 \pm 3^{*}$ & $111 \pm 6^{\dagger}$ & $127 \pm 5^{\ddagger}$ \\
\hline & 140 & $143 \pm 4^{*}$ & $113 \pm 11^{\dagger}$ & $138 \pm 6, \mathrm{NS}$ \\
\hline Protein in urine $(\mathrm{mg} / 24 \mathrm{~h})$ & 100 & $11 \pm 2^{*}$ & $62 \pm 9^{*}$ & $22 \pm 8^{*}$ \\
\hline \multirow[t]{2}{*}{ Urea in serum (mmol/L) } & 90 & $5.8 \pm 0.2^{\star}$ & $10.5 \pm 2.2^{\ddagger}$ & $7.2 \pm 1.0^{\dagger}$ \\
\hline & 140 & $5.3 \pm 0.5^{\star}$ & $32.6 \pm 9.4^{\star}$ & $7.3 \pm 0.6^{\star}$ \\
\hline \multirow[t]{2}{*}{ Creatinine in serum $(\mu \mathrm{mol} / L)$} & 90 & $21 \pm 2^{*}$ & $60 \pm 5^{\star}$ & $37 \pm 3^{*}$ \\
\hline & 140 & $24 \pm 1^{*}$ & $108 \pm 20^{*}$ & $35 \pm 4^{*}$ \\
\hline Grading histologic renal damage & 140 & $0.4 \pm 0.6^{\star}$ & $4 \pm 0^{*}$ & $1.3 \pm 0.5^{\ddagger}$ \\
\hline \multirow[t]{2}{*}{ 99mTc-DMSA (\%IA/kidney) } & 90 & $13.7 \pm 1.4^{*}$ & $6.4 \pm 2.4^{*}$ & $12.5 \pm 1.2, \mathrm{NS}$ \\
\hline & 140 & $18.1 \pm 1.9^{*}$ & $5.6 \pm 3.2^{*}$ & $14.3 \pm 1.1^{\dagger}$ \\
\hline 99mTc-MAG3 (\%IA/kidney, 2-6 min) & $100-120$ & $13.1 \pm 2.6^{\star}$ & $4.3 \pm 1.5^{\star}$ & $8.5 \pm 1.6^{\star}$ \\
\hline 99mTc-MAG3 (\%IA/cm³ cortex, 2-6 min) & $100-120$ & $6.9 \pm 1.9^{\star}$ & $2.6 \pm 1.3^{\ddagger}$ & $3.8 \pm 1.0^{\star}$ \\
\hline${ }^{111}$ In-DTPA (\%IA/kidney, 2-4 min) & $100-120$ & $4.7 \pm 1.0^{\dagger}$ & $2.1 \pm 1.4, \mathrm{NS}$ & $4.4 \pm 0.6, \mathrm{NS}$ \\
\hline \multicolumn{5}{|l|}{$\begin{array}{l}{ }^{*} P<0.0001 \\
{ }^{\dagger} P<0.001 . \\
{ }^{\ddagger} P<0.05 \\
\text { NS }=\text { not significant. } \\
\text { Quantification of retained renal radioac } \\
\text { activity was expressed as \% IA/kidney for }{ }^{11}\end{array}$} \\
\hline
\end{tabular}


renal damage at day 140 after therapy correlated with these observations. Both massive tubular and glomerular damage were observed in the kidneys of unprotected rats, whereas after lysine coinjection only minor abnormalities in tubular morphology were observed (Table 1).

Tubular damage beyond day 90 after therapy was confirmed by ${ }^{99 \mathrm{~m} T c-D M S A}$ static imaging (Table 1; Fig. 2). Renal ${ }^{99 \mathrm{~m} T c-D M S A}$ uptake in nontreated animals ranged from $14 \%$ to $19 \%$ injected activity (IA) per kidney, which significantly decreased to $2 \%-8 \%$ IA in PRRT-treated rats $(P<0.0001)$ and slightly decreased to $11 \%-15 \%$ IA in the kidneys of rats coinjected with lysine $(P<0.001)$. Ex vivo autoradiography confirmed these data.

Dual-isotope dynamic studies with ${ }^{111}$ In-DTPA and ${ }^{99 \mathrm{~m}} \mathrm{Tc}-\mathrm{MAG} 3$ in rats revealed a pattern in the time-activity curves similar to that in humans, with a peak of renal uptake for ${ }^{111} \mathrm{In}-\mathrm{DTPA}$ at 2-4 min and for ${ }^{99 \mathrm{~m} T c-M A G 3}$ at 2-6 min after administration. These peaks were followed by a decline of renal radioactivity, until most radioactivity was excreted at $20 \mathrm{~min}$ after injection and stabilized until $40 \mathrm{~min}$ after injection (Table 1; Fig. 3). Quantification of total renal ${ }^{99 \mathrm{~m} T c-}$ MAG3 radioactivity showed a mean peak activity of $13.1 \%$ IA in nontreated rats, whereas in PRRT rats only 4.3\%IA was detected. Lysine coadministration protected ${ }^{99 \mathrm{~m}} \mathrm{Tc}-\mathrm{MAG} 3$ extraction capacity of tubular cells after PRRT, because a significantly higher uptake $(P<0.0001)$ of $8.5 \%$ IA renal radioactivity was quantified in the PRRT + lysine group. Mean peak activities quantified only in cortical regions of the kidneys of the control, PRRT, and PRRT + lysine rats were $6.9 \%, 2.6 \%$, and $3.8 \% \mathrm{IA} / \mathrm{cm}^{3}$ cortex, respectively.
${ }^{111}$ In-DTPA imaging revealed a small but insignificant difference in peak activity between the control and PRRT + lysine groups ( $4.7 \%$ vs. $4.4 \% \mathrm{IA}$, respectively); however, ${ }^{111} \mathrm{In}$ DTPA uptake in the PRRT-only group was significantly less (2.1\%IA, $P=0.0003$ ), indicating that glomerular filtration was impaired after PRRT without kidney protection.

In clinical renography studies, patients are hydrated (i.e., $500 \mathrm{~mL}$ water by mouth $1 \mathrm{~h}$ before imaging) but are imaged with an empty bladder to stimulate efflux of radioactivity from the renal pelvis. Therefore, an extra group of control rats was imaged after extrahydration before the scan. The mean peak activities of ${ }^{99 m}$ Tc-MAG3 and ${ }^{111}$ In-DTPA remained unaltered, whereas the excretion of ${ }^{111}$ In-DTPA was unchanged and the efflux of ${ }^{99 \mathrm{~m} T c-M A G 3}$ was moderately enhanced during the last phase between 20 and $40 \mathrm{~min}$ after injection (data not shown).

\section{DISCUSSION}

In this small-animal SPECT study in rats, noninvasive renal imaging using ${ }^{99} \mathrm{~m}$ Tc-DMSA, ${ }^{99} \mathrm{~m}$ Tc-MAG3, and ${ }^{111}$ In-DTPA provided insight into both tubular and glomerular functions after high absorbed kidney radiation doses to monitor renal function as follow-up after PRRT. Simultaneous renography using dual-isotope dynamic imaging with ${ }^{99 m}$ Tc-MAG3 and ${ }^{111}$ In-DTPA was advantageous in restricting animal discomfort. Accurate quantification of renal radioactivity was obtained in short scans when 50 MBq of both ${ }^{99 m}$ Tc-MAG3 and ${ }^{111}$ In-DTPA were administered, leading to activity levels of $0.6-6 \mathrm{MBq}$ per kidney for ${ }^{99 \mathrm{~m}} \mathrm{Tc}$ and $0.4-2.5 \mathrm{MBq}$ for ${ }^{111} \mathrm{In}$. Compared with clinical
Control

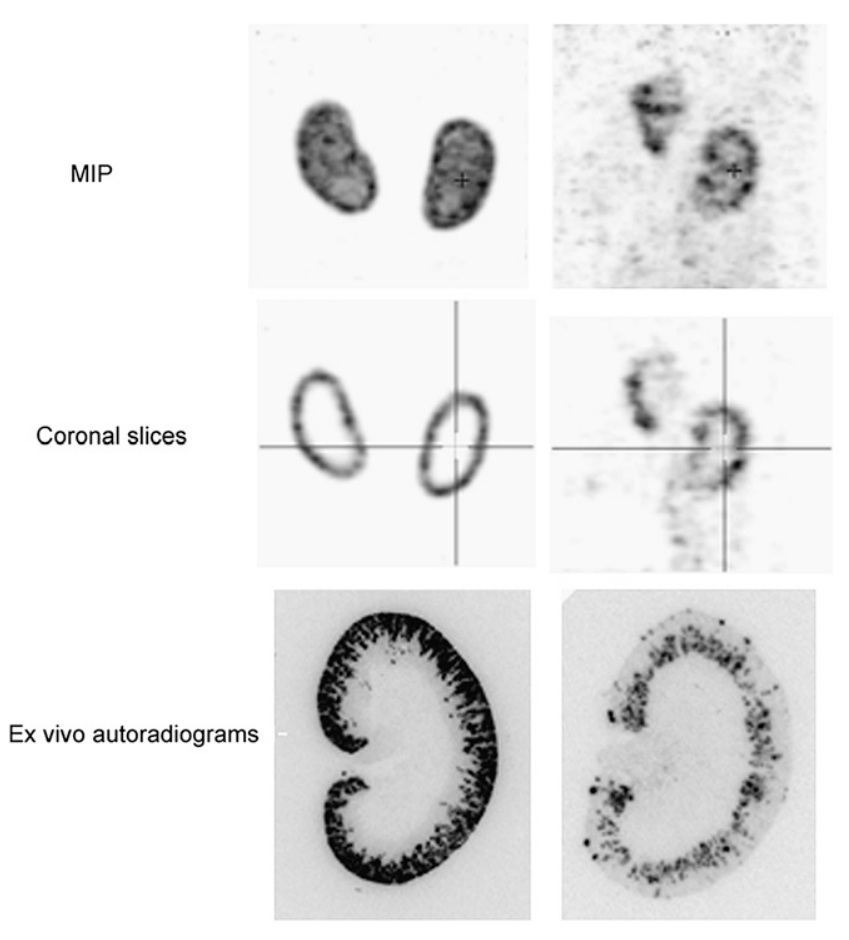

PRRT + Lys

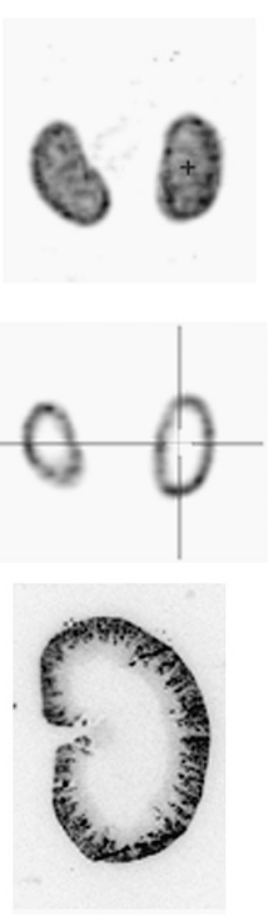

FIGURE 2. Typical images of maximumintensity projections and coronal slices of kidney after $99 \mathrm{mTc}$-DMSA scintigraphy performed at day 140 after therapy in nontreated control, PRRT-treated, or PRRT + lysine-treated rats. Ex vivo autoradiograms are of frozen kidney sections, prepared immediately after imaging. MIP = maximum-intensity projection. Lys = lysine. 
FIGURE 3. (A and B) Renography after injection of $50 \mathrm{MBq}$ of $99 \mathrm{mTC}-\mathrm{MAG} 3$, expressed as \%IA/kidney (A) and $\% \mathrm{IA} / \mathrm{cm}^{3}$ cortex (B). (C) Renography after injection of $50 \mathrm{MBq}$ of ${ }^{111} \mathrm{In}$-DTPA, expressed as \%IA kidney. (D) Typical example of a maximumintensity-projection dual-isotope image in control rats, 6 min after injection: $99 \mathrm{mTc}-$ MAG3 (left), ${ }^{111}$ In-DTPA (middle), and merged (right) images. Lys = lysine.

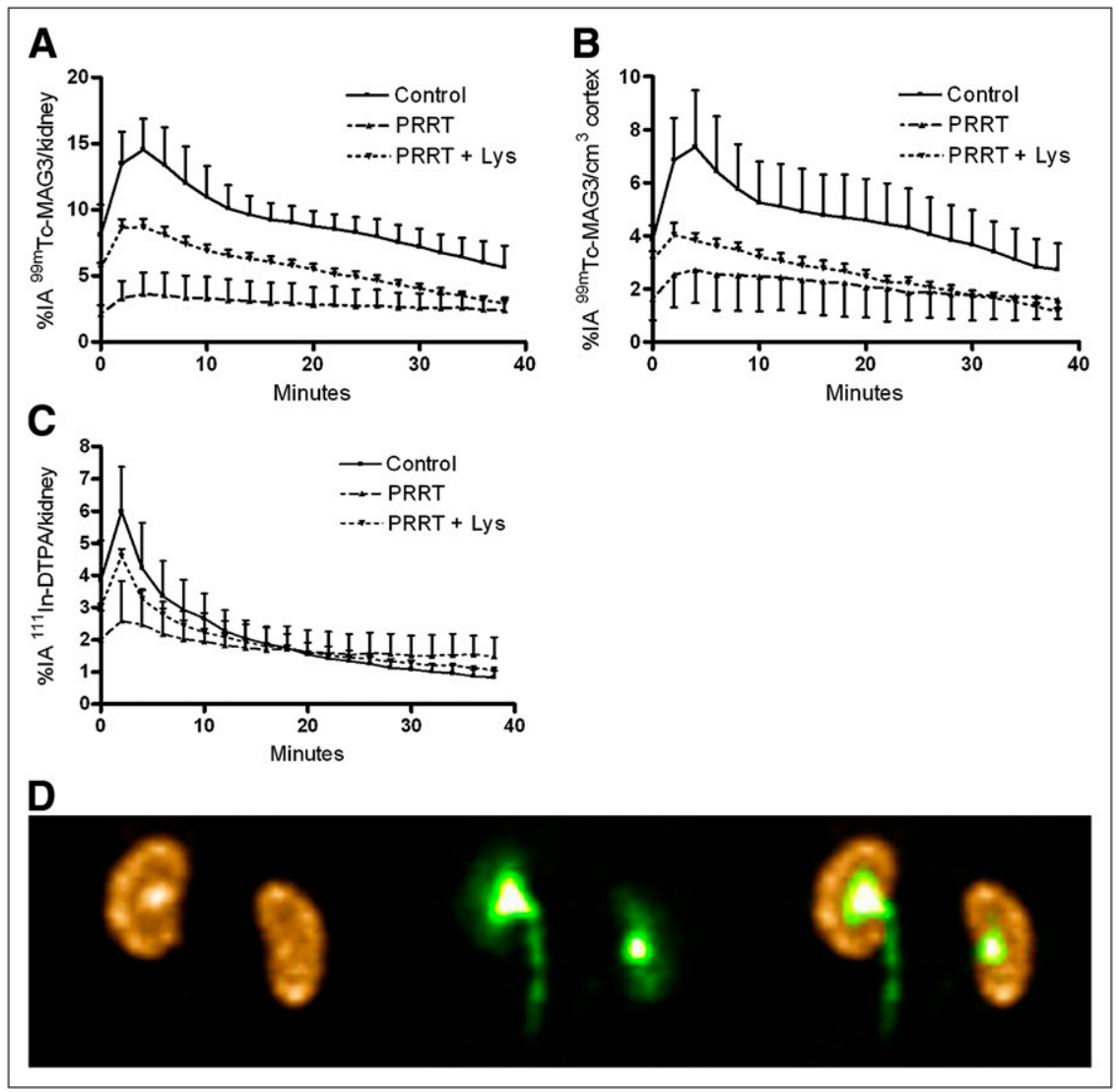

renography using frames of $15 \mathrm{~s}$ during a 20 -min scan, the 2-min frames in the rats were relatively long but had to be chosen because of the rotation time of the camera heads between projections. Recently, Bioscan developed highsensitivity apertures with enlarged pinhole diameters, offering improved time resolution. In the Linoview study (26), continuous dynamic SPECT was performed using 15-s frames for $30 \mathrm{~min}$ after injection of ${ }^{99 \mathrm{~m} T c-M A G 3}$, and the newly developed U-SPECT dedicated small-animal SPECT camera (Milabs) also will enable the acquisition of shorter frames than were used in this study (29).

Renographic ${ }^{99 m}$ Tc-MAG3 studies demonstrated more efficient elimination of the radioactivity from the kidneys in conscious mice than in anesthetized ones, indicating that the metabolic state of the animals is a confounding factor during dynamic renal imaging (26). Although the clearance rate of ${ }^{99 m}$ Tc-MAG3 and ${ }^{111}$ In-DTPA in our current study in anesthetized rats might not reflect the true physiologic state, the 3 groups of rats to be compared were imaged under equal, controlled conditions. Furthermore, the efflux of the radiopharmaceuticals from the kidneys to the bladder could be hampered by a filled bladder. The efflux of ${ }^{99 \mathrm{~m}} \mathrm{Tc}-$ MAG3 during the last phase of clearance was only slightly improved by extrahydration of the rats at $45 \mathrm{~min}$ before imaging (to stimulate emptying of the bladder), whereas the peak uptake of ${ }^{99 \mathrm{~m}} \mathrm{Tc}-\mathrm{MAG} 3$ and ${ }^{111} \mathrm{In}$-DTPA remained unchanged. Because of these extrahydration findings, hydration of the rats was not considered essential.

Previous experiments demonstrated that approximately $3 \%$ of the radiopeptide is reabsorbed per kidney $(16,17)-\mathrm{a}$ process that is mediated by the multiligand scavenger receptor megalin, which is expressed in the first part of the cortical proximal tubules $(30,31)$. Competition for the megalin receptor by lysine and radiolabeled octreotate, containing 1 lysine residue, might explain the reduction of renal retention found after coadministration with cationic amino acids $(10,13,31)$. In the current study, PRRT was administered using $460 \mathrm{MBq}$ of ${ }^{177} \mathrm{Lu}$-DOTA-Tyr ${ }^{3}$-octreotate. Long-term nephrotoxic effects were similar to those after the administration of $555 \mathrm{MBq}$ of PRRT, although the reduction of ${ }^{99 \mathrm{~m}} \mathrm{Tc}$-DMSA uptake was less severe (17). The aberrant localization pattern in PRRT kidney ${ }^{99 \mathrm{~m}} \mathrm{Tc}$-DMSA autoradiograms (Fig. 2) demonstrated that ${ }^{99 \mathrm{~m} T c-D M S A}$ was retained only in the nonmegalin receptor-expressing S3 part of the proximal tubules, located in the outer medulla, in contrast to the localization in control kidneys. Because ${ }^{111}$ In-DTPA renography demonstrated poor glomerular filtration in PRRT rats, ${ }^{99 \mathrm{~m} T c-D M S A}$ will not or will hardly be filtered and therefore not be reabsorbed in the proximal tubules. Therefore, peritubular extraction was the only route of ${ }^{99 m}$ Tc-DMSA retention in this part of the proximal tubules, as illustrated by the radioactivity localized in the 
outer medulla. The reduced level of retained ${ }^{177} \mathrm{Lu}$ in the kidneys of PRRT + lysine rats protected from affected glomerular filtration and megalin-mediated damage in the first convoluted part of the cortical proximal tubules as demonstrated by the unimpaired ${ }^{99 \mathrm{~m}}$ Tc-DMSA localization pattern. A role of megalin in the tubular reabsorption of ${ }^{99 \mathrm{~m}} \mathrm{Tc}-$ DMSA after glomerular filtration was confirmed by our observation that only $35 \%-55 \%$ of $99 \mathrm{~m}$ Tc-DMSA renal uptake was found when we imaged kidney-specific megalin-deficient mice (32), compared with wild-type mice (data not shown). Also in Clcn5 knockout mice, a model of Dent disease showing defective megalin- and cubilin-mediated endocytosis, heavily reduced ${ }^{99 m}$ Tc-DMSA uptake was observed, which matched results described in studies in patients with Dent disease or Fanconi syndrome $(25,26,33)$. Therefore, the conclusion drawn by Müller-Suur et al. that only peritubular extraction was responsible for renal ${ }^{99 \mathrm{~m}} \mathrm{Tc}-\mathrm{DMSA}$ uptake has been contradicted by these observations (22).

${ }^{99 m}$ Tc-MAG3 imaging showed no peak of tubular extraction in PRRT rats, either after total renal or after cortex-only ${ }^{99 m}$ Tc quantification; the latter method avoided confounding radioactivity already excreted into the renal pelvis (Fig. 3B). The clearance of ${ }^{99 m}$ Tc-MAG3 is not megalin or cubilin receptor-dependent, as discovered in Clcn5 knockout mice (26) and confirmed by us in megalin-deficient mice (data not shown), but is mediated by organic anion transport. This transport system is expressed in cells at the basolateral side of the convoluted proximal tubules and appeared to be inactive beyond $100 \mathrm{~d}$ after PRRT. Although lysine coadministration during PRRT significantly protects kidney function, ${ }^{99 m}$ Tc-MAG3 tubular extraction was clearly impaired when compared with control rats. It was proposed that ${ }^{99 m}$ Tc-DMSA was the optimal tracer to monitor and predict renal function $(3,34)$. However, the current data suggest that ${ }^{99 m}$ Tc-MAG3 renography is a more sensitive method to quantify loss of function of cortical proximal tubules than ${ }^{99 m}$ Tc-DMSA scintigraphy after PRRT. Unaffected ${ }^{99 \mathrm{~m}} \mathrm{Tc}-\mathrm{MAG} 3$ renography is one of the inclusion criteria before ${ }^{90} \mathrm{Y}$ - or ${ }^{177} \mathrm{Lu}$-based PRRT and is also used during follow-up at 1 center $(12,35)$, besides common glomerular filtration rate markers used at other medical centers $(8,14)$. Although further clinical validation is warranted, the introduction of routine ${ }^{99 \mathrm{~m}} \mathrm{Tc}-\mathrm{MAG} 3$ renography might be considered at more PRRT centers, especially in patients with compromised renal function. Also, patients receiving extra cycles of $\operatorname{PRRT}(7,8)$ are appropriate candidates for ${ }^{99 \mathrm{~m}} \mathrm{Tc}-\mathrm{MAG} 3$ follow-up when doses exceed the accepted safe limit of 23-27 Gy absorbed kidney radiation dose, adapted from external beam radiation, or the $37-$ to 45-Gy limit of the biologically equivalent dose on the kidney, taking into account the kidney mass and nonuniform localization of renal radioactivity $(36,37)$. The application of renal imaging using ${ }^{99 \mathrm{~m}}$ Tc-DMSA or ${ }^{99 \mathrm{~m} T c-M A G 3}$ can be extended to follow up renal proximal tubule function in other diseases when the kidney is the organ at risk $(26,38)$.

\section{CONCLUSION}

We demonstrated that quantitative renal imaging in rats is feasible using the NanoSPECT/CT small-animal camera, enabling the noninvasive monitoring of renal function over time. Both static ${ }^{99 m}$ Tc-DMSA scintigraphy and dynamic dual-isotope ${ }^{111}$ In-DTPA and ${ }^{99 m}$ Tc-MAG3 renography demonstrated that tubular and glomerular functions were affected beyond $90 \mathrm{~d}$ after PRRT with $460 \mathrm{MBq}$ of ${ }^{177} \mathrm{Lu}-$ DOTA-Tyr ${ }^{3}$-octreotate. Lysine coadministration preserved kidney function as monitored by ${ }^{111} \mathrm{In}$-DTPA and ${ }^{99 \mathrm{~m}} \mathrm{Tc}-$ DMSA. These results were consistent with the findings of kidney histology. Moreover, ${ }^{99 m}$ Tc-MAG3 renography seems to be a more sensitive marker of tubular function after PRRT than ${ }^{99 \mathrm{~m}}$ Tc-DMSA imaging, serum urea urinary protein content, or histology. Therefore, ${ }^{99 \mathrm{~m} T c-M A G 3}$ scintigraphy might gain a more prominent role in clinical PRRT.

\section{ACKNOWLEDGMENTS}

Collaboration within COST Action BM0607 contributed to stimulating discussions. The preparation of radiopharmaceuticals by Erik de Blois and other technicians of the Department of Nuclear Medicine, Erasmus MC, was greatly appreciated. We are grateful to Stuart Koelewijn for designing the illustration shown in Figure 1. This study was funded by KWF/NKB (Dutch Cancer Foundation), grant EMCR 2007-3758.

\section{REFERENCES}

1. Franc BL, Acton PD, Mari C, Hasegawa BH. Small-animal SPECT and SPECT/ CT: important tools for preclinical investigation. J Nucl Med. 2008;49:16511663.

2. Nuyts J, Vunckx K, Defrise M, Vanhove C. Small animal imaging with multipinhole SPECT. Methods. 2009;48:83-91.

3. Forrer F, Rolleman E, Schramm N, Krenning EP, de Jong M. Is it possible to predict renal function in small animals using a multi-pinhole SPECT system. Eur J Nucl Med Mol Imaging. 2007;34:1127-1128.

4. Kwekkeboom DJ, de Herder WW, van Eijck CH, et al. Peptide receptor radionuclide therapy in patients with gastroenteropancreatic neuroendocrine tumors. Semin Nucl Med. 2010;40:78-88.

5. Otte A, Herrmann R, Heppeler A, et al. Yttrium-90 DOTATOC: first clinical results. Eur J Nucl Med. 1999;26:1439-1447.

6. Cwikla JB, Sankowski A, Seklecka N, et al. Efficacy of radionuclide treatment DOTATATE Y-90 in patients with progressive metastatic gastroenteropancreatic neuroendocrine carcinomas (GEP-NETs): a phase II study. Ann Oncol. 2010; 21:787-794.

7. Forrer F, Uusijarvi H, Storch D, Maecke HR, Mueller-Brand J. Treatment with ${ }^{177} \mathrm{Lu}$-DOTATOC of patients with relapse of neuroendocrine tumors after treatment with ${ }^{90}$ Y-DOTATOC. J Nucl Med. 2005;46:1310-1316.

8. van Essen M, Krenning EP, Kam BL, et al. Salvage therapy with ${ }^{177}$ Lu-octreotate in patients with bronchial and gastroenteropancreatic neuroendocrine tumors. J Nucl Med. 2010;51:383-390.

9. Kunikowska J, Krolicki L, Hubalewska-Dydejczyk A, et al. Comparison between clinical results of PRRT with ${ }^{90}$ Y-DOTATATE and ${ }^{90} \mathrm{Y} /{ }^{177} \mathrm{Lu}$-DOTATATE [abstract]. Eur J Nucl Med Mol Imaging. 2009;36(suppl 2):S219.

10. Rolleman EJ, Valkema R, de Jong M, Kooij PP, Krenning EP. Safe and effective inhibition of renal uptake of radiolabelled octreotide by a combination of lysine and arginine. Eur J Nucl Med Mol Imaging. 2003;30:9-15.

11. Kwekkeboom DJ, Krenning EP, Lebtahi R, et al. ENETS Consensus guidelines for the standards of care in neuroendocrine tumors: peptide receptor radionuclide therapy with radiolabeled somatostatin analogs. Neuroendocrinology. 2009; 90:220-226. 
12. Prasad V, Fetscher S, Baum RP. Changing role of somatostatin receptor targeted drugs in NET: nuclear medicine's view. J Pharm Pharm Sci. 2007;10: $321 \mathrm{~s}-337 \mathrm{~s}$.

13. de Jong M, Rolleman EJ, Bernard BF, et al. Inhibition of renal uptake of indium111-DTPA-octreotide in vivo. J Nucl Med. 1996;37:1388-1392.

14. Bodei L, Cremonesi M, Ferrari M, et al. Long-term evaluation of renal toxicity after peptide receptor radionuclide therapy with ${ }^{90}$ Y-DOTATOC and ${ }^{177} \mathrm{Lu}-$ DOTATATE: the role of associated risk factors. Eur J Nucl Med Mol Imaging. 2008;35:1847-1856.

15. de Jong M, Breeman WA, Bernard BF, et al. $\left[{ }^{177} \mathrm{Lu}-\mathrm{DOTA}{ }^{0}, \mathrm{Tyr}^{3}\right]$ octreotate for somatostatin receptor-targeted radionuclide therapy. Int J Cancer. 2001;92: $628-633$.

16. Rolleman EJ, Krenning EP, Bernard BF, et al. Long-term toxicity of $\left[{ }^{177} \mathrm{Lu}-\right.$ DOTA $^{0}$, Tyr $^{3}$ ]octreotate in rats. Eur J Nucl Med Mol Imaging. 2007;34:219-227.

17. Forrer F, Rolleman E, Bijster M, et al. From outside to inside? Dose-dependent renal tubular damage after high-dose peptide receptor radionuclide therapy in rats measured with in vivo ${ }^{99 \mathrm{~m}} \mathrm{Tc}-\mathrm{DMSA}-\mathrm{SPECT}$ and molecular imaging. Cancer Biother Radiopharm. 2007;22:40-49.

18. Maisey M. Radionuclide renography: a review. Curr Opin Nephrol Hypertens. 2003;12:649-652.

19. Durand E, Prigent A. The basics of renal imaging and function studies. $Q \mathrm{~J} \mathrm{Nucl}$ Med. 2002;46:249-267.

20. van Luijk WH, Ensing GJ, Meijer S, Donker AJ, Piers DA. Is the relative ${ }^{99 \mathrm{~m}} \mathrm{Tc}-$ DMSA clearance a useful marker of proximal tubular dysfunction? Eur J Nucl Med. 1984;9:439-442.

21. Peters AM, Jones DH, Evans K, Gordon I. Two routes for ${ }^{99 \mathrm{~m}}$ Tc-DMSA uptake into the renal cortical tubular cell. Eur J Nucl Med. 1988;14:555-561.

22. Müller-Suur R, Gutsche HU. Tubular reabsorption of technetium-99m-DMSA. J Nucl Med. 1995;36:1654-1658.

23. de Lange MJ, Piers DA, Kosterink JG, et al. Renal handling of technetium-99m DMSA: evidence for glomerular filtration and peritubular uptake. J Nucl Med. 1989;30:1219-1223.

24. Forrer F, Valkema R, Bernard B, et al. In vivo radionuclide uptake quantification using a multi-pinhole SPECT system to predict renal function in small animals. Eur J Nucl Med Mol Imaging. 2006;33:1214-1217.

25. Lee BH, Lee SH, Choi HJ, et al. Decreased renal uptake of ${ }^{99 m} \mathrm{Tc}-\mathrm{DMSA}$ in patients with tubular proteinuria. Pediatr Nephrol. 2009;24:2211-2216.
26. Jouret F, Walrand S, Parreira KS, et al. Single photon emission-computed tomography (SPECT) for functional investigation of the proximal tubule in conscious mice. Am J Physiol Renal Physiol. 2010;298:F454-F460.

27. Shikano N, Kanai Y, Kawai K, Ishikawa N, Endou H. Transport of ${ }^{99 m}$ Tc-MAG3 via rat renal organic anion transporter 1. J Nucl Med. 2004;45:80-85.

28. Breeman WA, De Jong M, Visser TJ, Erion JL, Krenning EP. Optimising conditions for radiolabelling of DOTA-peptides with ${ }^{90} \mathrm{Y},{ }^{111} \mathrm{In}$ and ${ }^{177} \mathrm{Lu}$ at high specific activities. Eur J Nucl Med Mol Imaging. 2003;30:917-920.

29. van der Have F, Vastenhouw B, Ramakers RM, et al. U-SPECT-II: an ultra-highresolution device for molecular small-animal imaging. J Nucl Med. 2009;50: 599-605.

30. Christensen EI, Nielsen S, Moestrup SK, et al. Segmental distribution of the endocytosis receptor gp330 in renal proximal tubules. Eur J Cell Biol. 1995;66:349-364.

31. Melis M, Krenning EP, Bernard BF, et al. Localisation and mechanism of renal retention of radiolabelled somatostatin analogues. Eur J Nucl Med Mol Imaging. 2005;32:1136-1143.

32. Leheste JR, Melsen F, Wellner M, et al. Hypocalcemia and osteopathy in mice with kidney-specific megalin gene defect. FASEB J. 2003;17:247-249.

33. Kim SE, Cho JT, Lee DS, et al. Poor renal uptake of technetium-99m-DMSA and technetium-99m-MDP in a patient with Fanconi syndrome and near normal glomerular filtration rate. J Korean Med Sci. 1994;9:29-34.

34. Mitchell JR, Verweij M, Brand K, et al. Short-term dietary restriction and fasting precondition against ischemia reperfusion injury in mice. Aging Cell. 2010;9: 40-53.

35. Baum RP, Prasad V, Hommann M, Zachert C, Hoersch D. Treatment of metastasised neuroendocrine tumours with the radiolabeled somatostatin analogues Lu-177 or Y-90-DOTA-TATE: long term toxicity and survival in 543 patients [abstract]. Eur J Nucl Med Mol Imaging. 2009;36(suppl 2):S432.

36. Wessels BW, Konijnenberg MW, Dale RG, et al. MIRD pamphlet no. 20: the effect of model assumptions on kidney dosimetry and response-implications for radionuclide therapy. J Nucl Med. 2008;49:1884-1899.

37. Rolleman EJ, Melis M, Valkema R, et al. Kidney protection during peptide receptor radionuclide therapy with somatostatin analogues. Eur J Nucl Med Mol Imaging. 2009;37:1018-1031.

38. Rajic M, Ilic S, Vlajkovic M, et al. Radionuclide staging of renal function in type 1 diabetes mellitus. Ren Fail. 2007;29:685-691. 